\title{
« Histoire de bancs, parcours d'élèves »
}

Pour une lecture « configurationnelle » de la scolarisation à l'époque coloniale

Jean-Hervé Jézéquel

\section{QpenEdition}

1 Journals

Édition électronique

URL : http://journals.openedition.org/etudesafricaines/207

DOI : $10.4000 /$ etudesafricaines. 207

ISSN : 1777-5353

Éditeur

Éditions de l'EHESS

\section{Édition imprimée}

Date de publication : 1 janvier 2003

Pagination : 409-433

ISBN : 978-2-7132-1809-5

ISSN : 0008-0055

\section{Référence électronique}

Jean-Hervé Jézéquel, « « Histoire de bancs, parcours d'élèves » », Cahiers d'études africaines [En ligne], 169-170 | 2003, mis en ligne le 21 décembre 2006, consulté le 19 avril 2019. URL : http:// journals.openedition.org/etudesafricaines/207 ; DOI : 10.4000/etudesafricaines.207 


\section{Histoire de bancs, parcours d'élèves}

Pour une lecture « configurationnelle » de la scolarisation à l'époque coloniale

Partie intégrante de la «mission civilisatrice $»^{1}$, la politique scolaire des Français en Afrique occidentale française (AOF) a fait l'objet d'attentions répétées de la part des historiens (Autra 1956; Moumouni 1964 ; Bouche 1975 ; Désalmand 1983 ; Capelle 1990). Ces recherches ont d'abord souligné les contradictions internes et les hésitations des politiques coloniales. Elles ont ensuite mis à jour les décalages entre décideurs et acteurs en charge d'appliquer localement les politiques scolaires. En dépit de travaux de qualité, les études historiques consacrées à l'École en AOF ont privilégié l'offre scolaire au détriment des logiques de la scolarisation. En d'autres termes, l'histoire des bancs de classe a prévalu sur l'histoire de ceux qui les occupaient.

Si la question des réactions africaines face à l'École n'a pas fait l'objet d'études approfondies ${ }^{2}$, c'est peut-être parce qu'elle implique des processus historiques qui se laissent moins facilement appréhender à travers les archives coloniales conservées à Dakar ou Aix-en-Provence ${ }^{3}$. L'historiographie n'a certes pas totalement négligé cette question mais l'a souvent réduite à un scénario linéaire distinguant deux moments successifs de l'implantation scolaire: le rejet puis l'acceptation (Clignet \& Foster 1966: 4; Gérard 1997 : 99). «L'École du Blanc», perçue d'abord comme une contrainte,

1. Voir par exemple ConKLIN (1997: 75-106).

2. Il s'agit ici de lacunes dans le domaine historique. La rencontre entre sociétés africaines et École occidentale a en revanche fait l'objet de nombreuses études dans d'autres disciplines comme la sociologie, l'anthropologie ou les sciences de l'éducation. Pour un aperçu critique de cette littérature, voir BonINI (1996 : 22-27). On peut également voir la bibliographie donnée par GuTH (1990).

3. Les deux principaux centres d'archives pour l'histoire de l'AOF sont le Centre des Archives d'Outre-Mer d'Aix-en-Provence et les Archives nationales du Sénégal à Dakar. Les archives relatives à l'enseignement y sont conservées dans un état assez lacunaire. 
serait ensuite devenue le lieu privilégié de l'ascension sociale pour les populations africaines. Les seuls raffinements apportés à ce scénario univoque expliquent l'inégale progression de la scolarisation par l'ancienneté du contact avec l'Occident ${ }^{4}$, par certaines prédispositions « ethniques » (Clignet 1967 ; Martin 1972) $)^{5}$ ou encore par l'existence «d'obstacles structurels » comme l'islam (Khayar 1976).

Pourtant, les sociologues de l'éducation montrent que le rapport actuel des sociétés africaines à l'École occidentale s'inscrit dans des relations complexes et fluctuantes ${ }^{6}$. En se basant sur leurs résultats, l'historien est amené à son tour à questionner les schémas linéaires mis en place par l'histoire de la scolarisation en Afrique occidentale. Peut-on réellement réduire la période coloniale à une période de rejet massif de l'École lors de laquelle seule une minorité « occidentalisée » et «urbanisée » aurait su comprendre et profiter de l'institution?

L'objet de cet article est d'abord de souligner les limites des interprétations univoques et linéaires du processus de scolarisation à l'époque coloniale. Il propose ensuite une approche plus attentive aux interprétations et aux appropriations différenciées de l'École par les populations africaines au-delà d'un schéma binaire opposant le rejet à l'acceptation. Il ne s'agit pas de restituer l'ensemble des logiques et des processus en cours dans un cadre aussi large que celui de l'AOF mais, plus modestement, de dévoiler l'émergence d'un univers des possibles dans les relations entre sociétés africaines et École coloniale. Pour ce faire, nous nous proposons d'aborder la question de la scolarisation à travers l'étude d'un groupe particulier, les diplômés de l'École normale William-Ponty ${ }^{7}$ (ENWP). L'analyse de leurs itinéraires de scolarisation nous permettra de souligner combien les voies du

4. On oppose généralement une côte africaine précocement entrée en contact avec l'Occident à un intérieur demeuré plus longtemps hostile à la présence occidentale. Cette différence aurait conditionné des taux de scolarisation très inégaux engendrant des décalages régionaux qui se vérifient encore aujourd'hui (CLIGNET \& FOSTER 1966 ; SABATIER 1977).

5. Plus récemment, AyAndele (1992 : 227) explique l'engouement précoce des Ijebu (un sous-groupe yorouba du Sud Nigeria) pour l'École non seulement par certains attributs culturels partagés avec les autres groupes yoroubas («attributes of dynamism, adaptability, insatiable appetite, perspicacity and bootstrap philosophy ») mais aussi par leur perception plus claire des enjeux du moment : «[T]he Ijebu perceived early that the traditional non-literacy education that encouraged individual insularity was not geared to a modern society - one in which they would be integrated in the larger community of Nigeria in general and of Yorubaland in particular» (ibid. : 229).

6. Voir les résultats de la sociologie de l'éducation, entre autres le numéro spécial des Cahiers des Sciences humaines consacré à la scolarisation en Afrique et dirigée par M.-F. LANGE \& J.-Y. MARTIN (1995). Voir aussi Bonini (1996), GÉRARD (1997).

7. L'École Normale William-Ponty, longtemps située sur l'île de Gorée au Sénégal, a produit l'essentiel des instituteurs, des médecins et des commis ouest-africains avant les indépendances. Située au sommet de la hiérarchie scolaire, elle recrutait ses étudiants par voie de concours fédéral organisé dans toutes les écoles primaires supérieures (EPS) des colonies d'Afrique occidentale (JÉZÉQUEL 2002). 
recrutement scolaire sont non seulement complexes et multiples mais aussi étroitement dépendantes de dynamiques locales rarement appréhendées par «l'historiographie classique ».

\section{Les scénarios univoques de la scolarisation}

L'histoire de l'École en Afrique se trouve à la croisée de deux traditions historiographiques, l'une de langue anglaise, l'autre francophone. On n'enlève rien aux mérites respectifs de ces traditions en affirmant que l'histoire anglophone s'est volontiers faite sociale (Clignet \& Foster 1966 ; Sabatier 1977 ; Tibenderana 1983) quand l'histoire francophone restait largement institutionnelle (Bouche 1975; Désalmand 1983; Capelle 1990). Les travaux de Foster, consacrés au Ghana et à la Côte-d'Ivoire, se démarquent ainsi de l'historiographie francophone de la même époque par leur volonté de restituer la part africaine dans le développement de l'École. Plutôt que de privilégier l'offre scolaire ou l'étude des politiques coloniales d'éducation $^{8}$, Foster réintroduit l'action des sociétés locales au cœur du processus d'implantation et de transformation de l'École au Ghana (ancienne Gold Coast). Il montre notamment que le développement du système secondaire au Ghana, loin d'être la conséquence d'une politique volontariste du gouvernement colonial britannique, doit beaucoup aux efforts des premiers lettrés ghanéens. Plus largement, la naissance et la croissance de la demande scolaire en Gold Coast est, selon lui, le fruit des transformations de l'économie coloniale qui affectent à la fois la structure du marché de l'emploi (expansion des emplois liés au négoce ou à l'administration) et l'ensemble de la stratification sociale (dévalorisation progressive des anciens groupes dominants, attrait pour le statut de lettré, développement d'un idéal de "gentlemany »). Autrement dit, tout en soulignant le rôle de la demande africaine de scolarisation, Foster montre que le développement de l'École est étroitement lié à un ensemble de macro-transformations économiques et sociales qui s'inscrivent dans le sillage de la rencontre coloniale.

Cette approche que l'on peut qualifier de «développementaliste ${ }^{9}$ se révèle très enrichissante mais n'échappe cependant pas à certaines critiques. Mettant l'accent sur les caractéristiques communes des couches scolarisées,

8. Selon Foster (1965: 103-104), se limiter à l'histoire institutionnelle de l'École est une erreur si l'on veut comprendre l'implantation de l'École en Afrique subsaharienne : «Descriptions of educational goals or purposes as expressed by policy-makers are often an unproductive source of information on the processes of educational transfer [...]. The primary concern of the analyst must not be with formal policy but rather with the functional consequences of educational transfer [...]. Next, attention must be directed to the purposes for which the schools were being used by their African clientele and to the pattern of expectations generated among those who received formal education. »

9. Pour une présentation et une critique de l'École développementaliste, voir BAYART (1989 : 19-29). 
tant du point de vue social — prédominance des milieux « occidentalisés » — que géographique — prédominance des espaces urbains et côtiers précocement colonisés —, elle a produit l'image d'un processus de scolarisation uniforme et téléologique ${ }^{10}$. Cette perception imprègne encore aujourd'hui l'état des savoirs sur l'histoire de la scolarisation ${ }^{11}$. Son succès peut s'expliquer en partie par le fait qu'elle offre un cadre d'analyse très homogène et donc très commode pour appréhender de façon globale le processus de scolarisation en Afrique de l'Ouest.

D'autres travaux ont cependant cherché à compléter cette approche pour mieux expliquer l'inégal développement des niveaux de scolarisation entre les régions ou les groupes humains en Afrique de l'Ouest. Des chercheurs ont ainsi souligné l'existence de facteurs favorables ou, au contraire, d'obstacles «structurels » à la scolarisation comme les appartenances ethniques, sociales ou religieuses. Il n'est pas dans notre propos d'évoquer tous ces travaux mais, pour mieux cerner leur logique explicative, nous allons en présenter deux parmi les plus importants.

L'islam est souvent présenté comme un obstacle structurel à la diffusion de l'École coloniale. Ainsi, dans une étude intitulée «Le refus de l'École » consacrée à l'étude approfondie d'une région du nord-est tchadien, Issa H. Khayar (1976 : 83) montre bien comment « l'Islam, tel qu'il était pratiqué au Ouaddaï, se sentant [...] agressé, adoptait non seulement une attitude défensive mais se montrait agressif à son tour. Il combattait systématiquement toutes les valeurs véhiculées par l'École coloniale [...]. La majorité des Ouaddaïens en refusant l'école s'opposait en totalité à l'entreprise coloniale ». Jean Louis Triaud (1992: 149), spécialiste de l'islam en Afrique de l'Ouest, généralise l'opposition entre l'École française et l'islam quand il écrit : «Attachés à leur position de refus culturel qui a sans doute trouvé son expression la plus forte dans cette région que constitue le Ouaddaï au Tchad, les musulmans ont ignoré et combattu l'École française ${ }^{12}$. L'idée selon laquelle l'islam a gêné la diffusion des écoles françaises tient aujourd'hui du lieu commun. Pourtant, des travaux effectués sur d'autres terrains

10. Cette approche téléologique est notamment critiquée par les sociologues de l'École qui soulignent actuellement le retour en force des logiques de déscolarisation en Afrique subsaharienne (LANGE \& MARTIN 1995).

11. Ainsi GÉrARD (1997), dont les travaux soulignent avec beaucoup de finesse la complexité des rapports entre École et sociétés africaines contemporaines, est beaucoup moins attentif à cette même variété des attitudes dès lors qu'il traite de la période coloniale. Il reprend alors l'essentiel des thèmes de l'historiographie classique (alternance d'une phase de rejet et d'une phase d'engouement, méfiance des élites précoloniales et phénomène de substitution d'enfants de conditions serviles, etc.).

12. Dans la suite de cet article, Jean-Louis Triaud se montre cependant plus nuancé. Il souligne ainsi que le refus de l'École dont témoignent les musulmans se révèle ambigu. Certains leaders religieux sont en effet désireux de collaborer ou de s'accommoder avec le nouveau pouvoir occidental. 
ouest-africains nuancent cette opposition. Ainsi, Christian Coulon qui a étudié le Fuuta Tooro, région islamisée du Sénégal, affirme que les aristocraties toucouleurs, dont le pouvoir et la légitimité sont pourtant fortement liés à la religion musulmane, ont su se servir de l'École coloniale pour renforcer localement leurs positions de pouvoir ${ }^{13}$. Plus largement, en s'intéressant à l'évolution de la population scolarisée au Sénégal, on se rend compte que si, au XIX ${ }^{\mathrm{e}}$ siècle, les familles catholiques forment un groupe précurseur grâce à un enseignement essentiellement délivré par les missions chrétiennes ${ }^{14}$, au $\mathrm{Xx}^{\mathrm{e}}$ siècle, le développement de l'École publique a attiré une très large majorité de musulmans, reflétant logiquement la prédominance de l'islam dans la société sénégalaise. La présence à Ponty de ces musulmans est-elle seulement le résultat d'une contrainte coloniale exercée sur des familles musulmanes unanimement rétives à « l'École du Blanc »? Des Pontins sénégalais célèbres comme le romancier Abdoulaye Sadji ${ }^{15}$ ou l'ancien ministre Assane Seck $^{16}$ sont eux-mêmes fils ou petit-fils de responsables religieux et ont pourtant été envoyés volontairement dans une école que d'autres religieux rejetaient parfois avec une extrême virulence. Peuton réellement considérer leurs itinéraires comme autant d'exceptions à la règle ou faut-il envisager l'existence de liens moins univoques entre appartenance religieuse et logique de scolarisation à l'époque coloniale ?

De la même manière, d'autres études ont cherché à établir des liens entre statut social et scolarisation. Selon le sens commun, l'École coloniale aurait attiré les enfants d'esclaves plutôt que les fils de chefs ou de notables. En effet, les groupes dominants, méfiants envers «l'École du Blanc», auraient préféré envoyer les enfants de leurs serviteurs à la place de leur propre progéniture ${ }^{17}$. Cette représentation, solidement ancrée dans les représentations collectives, est confirmée par un certain nombre d'études. Ainsi, pour le Sénégal, Boubakar Niane (1987: 13) estime que les instituteurs sortis de Ponty sont « issus des couches non-aristocratiques ». Pour la Guinée, Claude Rivière (1975: 163) affirme que les élites du Fouta Djallon

13. Pour C. Coulon (1975 : 56), École et religion musulmane ne sont pas forcément antinomiques : «Tout en cherchant, par l'intermédiaire de l'école, à accéder aux nouvelles fonctions d'autorité, l'aristocratie tooroodo n'a pas cependant abandonné les éléments traditionnels de sa légitimité. » Tibenderana (1983) défend une idée semblable pour le Nord-Nigeria.

14. «Au début, l'enseignement à base religieuse des frères convenait à une société étroite, dominée par les habitants chrétiens » (Bouche 1975, t. 1: 139). Cela n'empêche pas la présence dans ces écoles missionnaires d'élèves issus des milieux islamisés. Ainsi, dans son autobiographie, l'ancien instituteur guinéen Fodé Lamine Touré raconte comment son grand-père, un marabout saint-louisien, est parti chercher fortune en Guinée au milieu du XIX ${ }^{\mathrm{e}}$ siècle. Il a par la suite renvoyé son premier fils au Sénégal pour qu'il y reçoive une «meilleure » éducation, en l'occurrence celle des missionnaires de Saint-Louis (Toure 1997 et entretien avec Fode Lamine Toure, Conakry [Guinée], août 2001).

15. Voir la biographie réalisée par son fils (SADJI 1997).

16. Entretien réalisé avec Assane Seck, Dakar, juillet 1999.

17. Voir par exemple, l'ouvrage de Moumouni (1964 : 55-56). 
(Guinée) sont restées, elles aussi, en marge de la scolarisation : «Les écoles françaises fixées assez tard dans quelques chefs-lieux comme Labé, Pita, Dabola, n'ont touché qu'une population fort réduite de serviteurs en majorité. » De nombreux travaux soulignent ainsi la réticence des élites précoloniales face à l'institution scolaire. Mais comment expliquer alors avec Éliane de Latour qu'en pays Mawri (Niger), les aristocraties locales aient été les premières à investir l'École ${ }^{18}$ ? Comment expliquer également que l'aristocratie du Fouta Tooro, pourtant proche dans son organisation de celle du Fouta Djallon, adopte, d'après Christian Coulon (1975 : 56), une attitude beaucoup plus conciliante envers « l'École du Blanc »? Comment expliquer enfin que parmi les Pontins sénégalais dont Niane a souligné les origines plutôt modestes, on trouve un petit-fils de Lat Dior, ancien Damel du Cayor, trois fils d'Ely Manel Fall, un chef de Province renommé, un fils d'El Hadj Mousse Diop, chef des Mourides de Dakar, plusieurs fils ou neveux de Cheikh Hamidou Kane, un des cadis les plus importants et les plus respectés de la région de Matam à l'époque coloniale ${ }^{19}$ ? Faut-il à nouveau considérer tous ces cas comme autant d'itinéraires marginaux qui n'invalident pas l'idée selon laquelle les élites précoloniales refusent massivement l'École française ?

Pour expliquer l'inégal développement de la scolarisation selon les régions ou les groupes humains, les recherches ont surtout tenté de dégager des macro-variables comme le degré de développement économique, l'ancienneté des contacts avec l'Occident, l'appartenance religieuse, ethnique ou sociale. Dans une perspective développementaliste, l'inégale dispersion de ces macro-transformations permettrait de comprendre les retards ou, au contraire, les accélérations du processus d'implantation de l'École occidentale selon les sociétés considérées. Sans renoncer aux résultats pertinents de ces travaux empiriques, il faut souligner les ambiguiités qui surgissent dès lors qu'on les confronte les uns aux autres. Plus largement, ce qui nous semble remis en cause, ce sont les approches globales et univoques du processus de scolarisation développées par ces études. Nous voudrions montrer que, dans un contexte ouest-africain peu homogène, les logiques de la scolarisation doivent être considérées moins comme des pratiques collectives déjà bien établies que comme des stratégies encore tâtonnantes traversant les appartenances sociales, religieuses ou ethniques.

18. «Les fils de chefs sont les premiers bénéficiaires de l'École française. Dans de nombreuses régions, les nobles, par crainte, substituent des esclaves à leurs enfants. En pays mawri, cela ne se fait pas [...]. Les jeunes Princes sont donc scolarisés et vont fournir les futurs commis de l'État colonial » (DE LATOUR 1987 : 170-171).

19. Ces informations sont issues d'un fichier prosopograhique constitué essentiellement à partir des registres de l'École normale William-Ponty (série O, Archives nationales du Sénégal, Dakar). 


\section{La diversité des itinéraires de scolarisation}

En étudiant un groupe particulier de scolarisés, en l'occurrence les diplômés de l'École normale William-Ponty ${ }^{20}$, nous allons montrer que les logiques de la scolarisation sont, dès l'époque coloniale, plus diverses et complexes que ne le laisse entrevoir l'historiographie.

Selon les approches de type développementaliste, la scolarisation aurait fortement favorisé les milieux urbains sur les sociétés rurales, les « sociétés côtières » précocement colonisées sur celles de «l'intérieur ${ }^{21}$. En effet, l'École apparaîtrait comme une clé d'accès privilégiée à l'économie salariée qui se développe plus précocement dans les espaces urbains et côtiers. L'étude prosopographique de l'origine des Pontins ne confirme pas complètement cette hypothèse : d'une colonie à l'autre, on voit apparaître des géographies distinctes du recrutement de l'élite scolaire (voir tableau I).

Tableau I. - Origine géographique des Pontins du SÉnégal, Dahomey, Soudan, Guinée et Haute-Volta ENTRE 1906 et 1948 (EN \%)

\begin{tabular}{lccccc}
\hline & Sénégal & Dahomey & Soudan & Guinée & Haute-Volta \\
\hline $\begin{array}{l}\text { Village ou bourg } \\
\text { (moins de 2 000 habitants) }\end{array}$ & 9 & 11 & 29 & 43 & 59 \\
\hline $\begin{array}{l}\text { Petite et moyenne } \\
\text { agglomération (moins de }\end{array}$ & 27 & 24 & 41 & 28 & 24 \\
\begin{tabular}{l}
10 000 habitants) \\
\hline $\begin{array}{l}\text { Agglomération de plus de } \\
10000 \text { habitants }\end{array}$
\end{tabular} & 63 & 65 & 30 & 29 & 17 \\
\hline
\end{tabular}

Source : fichier prosopographique, in Jézéquel 2002.

Le tableau ci-dessus fait ressortir des oppositions très marquées entre les colonies ouest-africaines $^{22}$ : la majorité des Pontins originaires de Haute-Volta

20. Au cours de nos différents séjours d'enquêtes en Afrique de l'ouest, nous avons pu réaliser une centaine d'entretiens avec d'anciens Pontins. Cela nous a permis de reconstituer avec précision les itinéraires socioprofessionnels de ces anciens instituteurs diplômés de l'École normale William-Ponty (JÉZÉQueL 2002).

21. «Popular pressures for schooling tend to expand more rapidly in precisely those areas where economic change is already under way and where elaboration of the occupational structure is more marked. This is particularly true in urban centers where the development of an exchange economy and the emergence of new type occupations create a demand for schooling. [...] » (CLIGNET \& FosTER $1966: 5$ ).

22. Il est important de noter que ces colonies ne sont pas également représentées à Ponty. Les contingents de Pontins par colonies sont fortement inégaux. Ainsi, à elles seules, les colonies du Soudan et du Sénégal fournissent près de la moitié des élèves passés par l'École normale entre 1904 et 1947. Toutefois, ce nombre d'élèves ne prend son sens que si on le rapporte à la population totale de chaque colonie. Les hiérarchies se recomposent alors : le Sénégal et le Dahomey se détachent nettement avec respectivement 0,27 et 0,33 Pontins pour 1000 habitants. 
est formée de ruraux alors qu'à l'opposé, la majorité des Pontins sénégalais et dahoméens vient des grandes villes. Cela se comprend aisément si l'on se rappelle qu'à l'époque coloniale, le développement des villes est plus important au Sénégal et au Dahomey qu'en Haute-Volta. Des territoires comme le Soudan français ou la Guinée présentent quant à eux des situations plus ambiguës : si la majorité des Pontins est d'origine «urbaine » (avec toute l'imprécision que revêt ce terme après une codification forcément appauvrissante ${ }^{23}$ ), il n'en reste pas moins que près d'un tiers (de $29 \%$ au Soudan jusqu'à $43 \%$ en Guinée) vient de petits villages ou de bourgs sans infrastructure administrative coloniale. De plus à l'inverse du Sénégal, les Pontins du Soudan viennent plutôt des villes moyennes que des grandes villes $^{24}$. Or, ces villes secondaires de la colonie du Soudan comme Nioro, Kita ou Bandiagara conservent un caractère rural encore très marqué notamment dans la composition socioprofessionnelle de la population. Contrairement à ce qu'affirme le courant développementaliste, l'élite scolaire ouestafricaine n'est pas forcément originaire des zones urbaines.

On peut faire la même remarque en ce qui concerne la prédominance des populations côtières parmi les premiers scolarisés. Si l'on compare, par exemple, l'origine des Pontins guinéens et dahoméens, la différence est frappante :

Tableau II. - Répartition des Pontins guinéens et Dahoméens EN FONCTION DU CERCLE D'ORIGINE

\begin{tabular}{l|rr|cr}
\hline & \multicolumn{2}{|c|}{ Dahomey } & \multicolumn{2}{c}{ Guinée } \\
& Nombre & Part & Nombre & Part \\
\hline $\begin{array}{l}\text { Pontins originaires } \\
\text { d'une région côtière }\end{array}$ & 201 & $72 \%$ & 46 & $19 \%$ \\
$\begin{array}{l}\text { Pontins originaires } \\
\text { d'une région intérieure }\end{array}$ & 73 & $26 \%$ & 177 & $72 \%$ \\
Inconnus & 6 & $2 \%$ & 22 & $9 \%$ \\
\hline
\end{tabular}

Source : fichier prosopographique, in Jézéquel 2002.

Le Soudan, la Guinée et la Côte-d'Ivoire (Haute-Volta incluse) se tiennent avec une même moyenne de 0,12 Pontin pour 1000 habitants proches de la moyenne pour l'ensemble de l'AOF $(0,14)$. Selon Sabatier, la sur-représentation du Sénégal et du Dahomey s'explique par les transformations macro-sociales et macroéconomiques précoces de ces deux colonies, dont le taux d'urbanisation et le contact avec l'Occident sont plus anciens qu'ailleurs. Cette explication ne doit cependant pas conduire à négliger l'étude approfondie des autres contingents de Pontins comme le fait Sabatier qui prend prétexte du manque de données. Elle s'empêche par là même de constater l'existence de logiques de scolarisation très différentes dans des colonies comme le Soudan, la Côte-d'Ivoire et la HauteVolta. Or, posé en termes bruts, le nombre de Pontins soudanais et ivoiriens est loin d'être négligeable en regard du contingent sénégalais (environ 440 Soudanais et 330 Ivoiriens contre 550 Sénégalais).

23. Dans un cadre aussi large que l'Afrique occidentale française, il n'a pas été possible d'adopter une typologie plus fine sur l'origine géographique de Pontins (JÉZÉQUEL 2002).

24. En 1934, on compte 7 villes de plus de 10000 habitants au Sénégal contre 4 seulement au Soudan et 5 en Guinée. Sources : Annuaire statistique de l'Afrique occidentale française, 1933-1934, Coulomiers, Imprimeries Brodard et Taupin, 1936. 
Si le cas dahoméen offre bien l'image d'une élite scolaire massivement originaire des régions côtières, il n'en va pas de même pour la Guinée où près des trois-quarts des Pontins viennent des régions intérieures. On peut compléter ce premier exemple à l'aide du tableau III qui évalue la dispersion ou la concentration des lieux d'origine des Pontins dahoméens et togolais d'une part, et ivoiriens d'autre part. Il montre notamment que le recrutement des Pontins ivoiriens est géographiquement plus éclaté que celui des Pontins togolais et dahoméens.

\section{TABLEAU III. — L'INÉGALE DISPERSION DES ORIGINES GÉOGRAPHIQUES DES PONTINS EN Côte-D'Ivoire ET AU TOGO/DAHOMEY}

\begin{tabular}{l|c|c}
\hline & $\begin{array}{c}\text { Nombre de localités } \\
\text { d'où sont originaires } \\
\text { un ou plusieurs Pontins }\end{array}$ & $\begin{array}{c}\text { Nombre de Pontins } \\
\text { dont on connaît l'origine }\end{array}$ \\
\hline $\begin{array}{l}\text { Côte-d'Ivoire } \\
\text { Dahomey et Togo }\end{array}$ & $\begin{array}{r}169 \text { lieux de naissance différents } \\
56 \text { lieux de naissance différents }\end{array}$ & $\begin{array}{r}348 \text { cas recensés } \\
352 \text { cas recensés }\end{array}$ \\
\hline
\end{tabular}

Source : fichier prosopographique, in Jézéquel 2002.

Cette courte analyse prosopographique n'a pas pour vocation de nier la surreprésentation des urbains ou des côtiers parmi l'élite scolarisée. Elle suggère simplement que la composition de l'élite scolaire est plus complexe qu'il n'y paraît. Les Pontins d'origine rurale forment en effet un groupe non négligeable et parfois majoritaire dans certains territoires. Plutôt que de négliger ces données ou d'interpréter les inégalités en termes de retard de certaines colonies dans le processus d'urbanisation, il faut comprendre que la scolarisation obéit à des logiques plurielles qui s'ancrent, pour partie, dans les dynamiques locales des sociétés rurales.

En poursuivant l'analyse prosopographique, on peut d'ailleurs s'interroger sur l'existence de milieux privilégiés dans lesquels se recruteraient les élites scolarisées en général et les Pontins en particulier. Selon l'approche développementaliste, l'élite scolarisée est très majoritairement issue des milieux déjà en partie occidentalisés ${ }^{25}$. Selon ces travaux, la proximité socioprofessionnelle avec les colonisateurs ou les missionnaires et l'occidentalisation des modes de vie et des aspirations seraient à l'origine d'une «proto-élite » (Kipkorir 1973; Sabatier 1977) qui constituerait elle-même le milieu d'origine des élites lettrées autochtones. En interrogeant l'origine des Pontins selon une démarche prosopographique, on peut tester cette hypothèse dans le cadre de l'AOF.

25. «[...] A disproportionate share of Ponty graduates come from partly westernized homes » (SABATIER 1976: 416-417). 
Tableau IV. - Origine des Pontins SElon la PROFession du PÈRE

\begin{tabular}{lcr}
\hline & Nombre & $\%$ \\
\hline Agriculteurs et artisans & 221 & 33,1 \\
Chefferies et notabilités & 90 & 13,5 \\
Auxiliaires ou fonctionnaires coloniaux & 150 & 22,4 \\
Commerçants, boutiquiers et traitants & 149 & 22,3 \\
Pères européens ou levantins & 58 & 8,7 \\
\hline Total & 668 & 100,0 \\
\hline
\end{tabular}

Source : fichier prosopographique, in Jézéquel, 2002.

Le tableau ci-dessus présente à première vue un groupe aux origines relativement éclatées. Certes, la part de Pontins dont le père exerce une profession en relation ${ }^{26}$ avec les milieux européens est incontestablement forte. En effet, un père de Pontin sur cinq exerce un emploi salarié directement lié au service colonial. Cette proportion prend toute son ampleur quand on sait que l'activité salariée ne concerne alors que 1 à $2 \%$ de la population totale de l'AOF: «En 1936, le nombre des salariés d'AOF se limitait à 167 000, soit à peine plus de $1 \%$ de la population » (Coquery-Vidrovitch 1992 : 33). D'un autre côté, un tiers des pères de Pontins exerce une activité agricole ou artisanale sans rapport direct avec la présence européenne ; une même proportion est concernée par des activités commerciales. Aucune catégorie sociale n'occupe donc de position de monopole à l'École WilliamPonty.

\section{Les logiques de la scolarisation à l'époque coloniale}

L'analyse prosopographique a mis en exergue la diversité des logiques de scolarisation à l'époque coloniale ${ }^{27}$. Cette diversité va à l'encontre des analyses univoques du fait scolaire en Afrique de l'Ouest. Ces analyses ont en effet privilégié le rôle de «macrovariables » comme le degré d'urbanisation ou l'appartenance sociale, ethnique et/ou religieuse dans l'étude de la scolarisation. Or ces approches «par le haut» ne sont pas forcément les plus

26. Par ce terme, nous entendons un contact prolongé et répété dans le temps : un traitant qui vend ou achète ses produits aux maisons européennes ; le fonctionnaire africain qui travaille au quotidien à la résidence de l'administrateur; le chef de canton, nommé par l'administration coloniale et qui doit régulièrement lui rendre des comptes pour les impôts ou les tournées de recrutement. On se rend compte ainsi combien ce contact professionnel avec les milieux européens emprunte des voies très diverses.

27. Cette diversité des itinéraires de scolarisation, vérifiée ici dans le cas des diplômés de l'ENwP, caractérise a fortiori l'ensemble des populations scolarisées à l'époque coloniale. 
pertinentes ${ }^{28}$. En effet, face à une institution «neuve » comme l'École coloniale, il est illusoire de vouloir cerner d'emblée des stratégies collectives reflétant les appartenances sociales, religieuses ou ethniques. En effet, les comportements et les stratégies des individus face à cette institution «neuve » sont encore peu structurés, en voie d'émergence, et traversent les identités et les appartenances collectives. Dès lors, plutôt que de privilégier arbitrairement un niveau d'analyse, il faut plutôt tenter de les repenser ensemble pour reconstituer le plus finement possible la «trame des déterminations ».

\section{Des registres de causalité pluriels}

L'examen des récits de vie recueillis sur le terrain permet ainsi de montrer comment les itinéraires de scolarisation à l'époque coloniale sont le résultat de déterminations plurielles qui jouent à différents niveaux de l'analyse et qu'il serait vain de vouloir isoler ou penser séparément. Nous nous proposons de le montrer à travers l'itinéraire de C. B., un instituteur originaire de Bobo-Dioulasso sur lequel nous disposons de plusieurs témoignages ${ }^{29}$.

Né à Bobo-Dioulasso, dans l'ouest de l'ancienne Haute-Volta, C. B. est le fils d'un commerçant qui travaille pour le compte d'une compagnie de traite française. Sans avoir été lui-même scolarisé, le père de C. B. a su acquérir les rudiments de français et de comptabilité indispensables à sa fonction d'intermédiaire entre les maisons de commerce françaises et les populations africaines. Conscient des avantages et des opportunités professionnels qu' offre la maîtrise de la langue du colonisateur, il a volontairement placé l'ensemble de ses fils à l'école. Ce premier niveau du récit souligne l'importance des dynamiques macro-sociales dans la scolarisation : la décision du père de C. B. s'inscrit dans un processus plus large, celui du développement d'une économie coloniale. Cette dernière a transformé le marché de l'emploi et généré de nouvelles opportunités professionnelles qui expliquent l'itinéraire scolaire de C. B. dans la logique des travaux développés par Foster (1965).

28. Cela est peut-être d'autant moins pertinent puisque ici on traite de la scolarisation comme d'un phénomène historique émergent face auquel les acteurs sociaux élaborent des stratégies et des comportements encore tâtonnants et incertains.

29. Lorsque nous avons réalisé nos enquêtes, C. B. était décédé depuis plusieurs années. Il avait toutefois accordé un entretien à une radio locale et nous avons pu nous procurer une copie de l'émission dans laquelle il se livrait à l'exercice autobiographique. Ayant contacté la famille de cet instituteur, nous avons également pu réaliser deux entretiens, l'un avec son frère cadet, ancien chauffeur devenu imam d'une mosquée de quartier, et l'autre en dioula avec sa dernière épouse. En complétant notre enquête par quelques documents d'archives trouvés à Dakar et Bobo-Dioulasso, nous avons pu réunir et vérifier un ensemble d'informations assez satisfaisant pour reconstituer l'itinéraire de C. B. 
Le même récit de vie révèle cependant d'autres dynamiques lorsqu'on se montre attentif à des dynamiques plus locales. Bien que C. B. soit luimême né à Bobo-Dioulasso, la seconde ville de Haute-Volta, sa famille est originaire du Sénégal. Son père est un traitant toucouleur de la région de Saint-Louis. Après avoir travaillé à Kayes et Médine (Soudan français), il se rend à Bobo-Dioulasso au début du siècle pour y tenir une boutique. Ils y retrouvent d'autres Sénégalais venus eux aussi s'installer à Bobo-Dioulasso au début du siècle. Traitants pour la plupart, ils entretiennent entre eux des liens d'affaires et de voisinage très étroits ${ }^{30}$. Ces Sénégalais se distinguent du reste de la population par un ensemble de pratiques spécifiques (occupation particulière de certains quartiers de Bobo-Dioulasso ${ }^{31}$, pratiques alimentaires et vestimentaires, habitudes religieuses, etc.). Ils constituent, à BoboDioulasso, une petite communauté qui cherche autant à s'intégrer qu'à préserver sa spécificité et ses privilèges. Pour marquer la distance qui les sépare des «populations voltaïques », ce groupe de Sénégalais a précocement développé des stratégies de distinction. Dans sa thèse sur les Européens et assimilés de Basse Côte-d'Ivoire, Alain Tirefort (1979) a montré comment les Sénégalais expatriés en Côte-d'Ivoire ont développé de semblables stratégies de distinction, notamment par l'intermédiaire de clubs et de lieux de sociabilités spécifiques ${ }^{32}$. À Bobo-Dioulasso, dans une ville où la population locale est majoritairement hostile à 1 'école ${ }^{33}$, la scolarisation des

30. Entretien avec A. Diakhité, Bobo-Dioulasso. Ancien commerçant, lui-même fils d'un traitant « sénégalais » venu s'installer à Bobo-Dioulasso à l'instar du père de C. B., A. Diakhité nous a entretenu des liens qui unissaient les différentes familles sénégalaises dès les débuts de l'époque coloniale. Les archives confirment que ces liens se sont étendus au domaine politique puisque dans les années 1940, les «Sénégalais » forment un groupe à part dans l'effervescence politique qui anime alors la ville de Bobo-Dioulasso (Archives du CNRST à Ouagadougou, Série B, IV-2 ; cercle de Bobo-Dioulasso, 1945-1950).

31. Pour les pratiques foncières et les stratégies d'accumulation des Sénégalais de Bobo-Dioulasso, voir par exemple FourCHARD (2001).

32. L'autobiographie de Birago Diop constitue une source supplémentaire d'informations sur les Sénégalais de Bobo-Dioulasso. Dans le second tome de ses mémoires, cet ancien vétérinaire sénégalais se souvient en effet d'avoir fréquenté les groupes sénégalais de Bobo-Dioulasso lors de son affectation dans la ville vers 1945. Son premier logeur fut Saada Cissé, commerçant toucouleur originaire du Fouta Toro et personnalité de premier plan parmi ceux que Birago Diop appelle lui-même "les miens sénégalais de la "Diaspora" » (DIOP 1982: 58). Il décrit ainsi la maison de son hôte: "Quand je rentrai chez Sada Cissé, midi largement passé, la véranda de la maison était pleine de notabilités soudanaises, guinéennes, sénégalaises, amies du maître de maison, dont beaucoup sinon tous avaient été ou étaient encore ses obligées. Je fis la connaissance, entre autres de Maky Sy, interprète et... pâtissier, Medoune Diop, Abdou Kane Diop, Lassana Kaba, doyen des Guinéens. Et de Samba Diop, Sénégalais, ancien boxeur qui tenait un bistrot non loin de la maison » (ibid. : 61).

33. Les rapports scolaires rédigés par les directeurs successifs de l'école de BoboDioulasso entre 1904 et 1920 soulignent tous la franche hostilité des populations autochtones à l'égard de l'institution scolaire (Archives nationales de France, $\mathrm{J}-49)$. 
enfants s'avère pour les traitants sénégalais un moyen de se distinguer et de conserver leur position d'intermédiaires privilégiés entre les Français et la «population indigène ». La scolarisation de C. B. ne serait donc pas seulement la conséquence des macro-transformations affectant le marché du travail et plus globalement les structures économiques en Afrique de l'Ouest. Intégrée aux stratégies de reproduction d'une identité «sénégalaise », elle répond aussi à des logiques intermédiaires ou méso-sociales de reproduction et de distinction ${ }^{34}$.

En centrant enfin notre attention sur l'unité familiale construite autour de la figure paternelle, premier arrivé à Bobo-Dioulasso, on peut saisir un troisième et dernier niveau de détermination, de nature micro-sociale celuici. Tous les frères de C. B. sont nés comme lui à Bobo-Dioulasso et sont également allés à l'école où ils ont effectué des parcours très inégaux. En conséquence, leurs itinéraires professionnels s'avèrent également très disparates. A côté de C. B., l'aîné, qui a intégré un cadre prestigieux de l'administration coloniale, seul Malick ${ }^{35}$, greffier au tribunal et diplômé de l'École normale William-Ponty (section administration) a connu une carrière aussi réussie. À l'inverse, tous leurs cadets semblent avoir eu moins de chance dans les études et dans leur vie active : Tidiani est devenu chauffeur, Ibrahima a intégré le cadre local des Travaux Publics, Birama a trouvé une place de menuisier à Bouaké (Côte-d'Ivoire), tout comme Adama devenu ensuite chauffeur puis commis des Postes. Or, cette diversité des itinéraires professionnels est probablement liée aux différents statuts dont jouissent les enfants au sein de la famille. En effet, le père de C. B. a pris pour première épouse une femme toucouleur d'ascendance noble, originaire comme lui de la vallée du Fleuve Sénégal et venue avec son père à Bobo-Dioulasso. Les deux premiers garçons, C. B. et Malick, sont les enfants de cette femme d'ascendance noble. Par la suite, le père de C. B. a acheté plusieurs épouses sur le marché de Bobo-Dioulasso ${ }^{36}$. Les autres frères de C. B. sont les enfants de ces femmes de condition servile. On peut alors penser que le père de $\mathrm{C}$. B. a plus particulièrement favorisé les études de ces deux premiers garçons qui jouissent d'un meilleur statut au sein de la famille en les poussant vers la haute fonction administrative. À l'inverse, il a interrompu les études des cadets (de mères de condition servile) parce qu'il avait besoin d'eux : deux de ses fils ont ainsi cessé leurs études pour servir de chauffeurs dans l'entreprise familiale. Cet exemple suggère que le statut et le rang des individus sont certainement des éléments à prendre en compte dans

34. Encore aujourd'hui, les membres de la famille de C. B. se disent «sénégalais » bien que n'ayant guère de contact avec leur pays d'origine. Entretien informel avec les enfants dans la concession familiale à Bobo-Dioulasso.

35. Les prénoms ont été modifiés pour garantir l'anonymat des personnes.

36. Malgré l'abolition de l'esclavage, le marché de Bobo-Dioulasso semble toujours être un lieu de vente d'esclaves au début $\mathrm{du} \mathrm{Xx}^{\mathrm{e}}$ siècle. Ce commerce a été très actif dans la région ainsi que dans le Kénédougou voisin dont les razzias ont particulièrement touché les populations locales. 
l'explication des itinéraires de scolarisation ${ }^{37}$. À une époque où la scolarisation ne constitue pas encore une pratique sociale partagée, des microdéterminations comme le statut personnel ou le rang des enfants dans la famille influencent fortement les trajectoires scolaires.

L'itinéraire de C. B. met en lumière les différents niveaux de causalité qui ont favorisé sa scolarisation. Celle-ci résulte de l'entrecroisement de déterminations liées aux macro-transformations économiques, aux stratégies de reproductions des groupes sociaux ainsi qu'au statut personnel des individus dans leur groupe d'origine. Au lieu de privilégier un niveau de détermination sur un autre, il faut envisager la scolarisation comme le résultat de la conjugaison de leur influence. La reconstitution d'itinéraires permet ainsi d'enrichir les approches classiques et linéaires de la scolarisation. Elle offre en outre l'avantage de dépasser la question des obstacles structurels à la scolarisation en soulignant, comme nous allons le voir, le caractère « modulaire $»^{38}$ des déterminations.

\section{Pour une approche configurationnelle de la scolarisation}

À l'époque coloniale, les logiques de la scolarisation obéissent non seulement à plusieurs niveaux ou plusieurs registres de déterminations mais le sens de chacune de ces déterminations n'est lui-même pas figé. Dans une perspective configurationnelle $\mathrm{e}^{39}$, il ne se révèle que lorsque l'on réinsère chaque détermination dans la trame des variables avec laquelle elle interagit. Des facteurs tels que l'appartenance religieuse ou le statut social peuvent donc, selon les cas, favoriser ou, au contraire, entraver la scolarisation. On peut souligner l'impact différencié des déterminations à travers l'étude des Tall, une famille prestigieuse de l'ancien Soudan français.

37. On retrouve simplement ici les processus mis en évidence par les sociologues de l'éducation en ce qui concerne les pratiques contemporaines de scolarisation en Afrique subsaharienne (LANGe \& MARTIN 1995 ; GÉRARD 1997).

38. Par modulaire ou modularité, nous entendons le fait qu'un facteur de causalité a un impact différencié en fonction de la trame des déterminations dans laquelle il s'insère et avec laquelle il interagit.

39. Maurizio GRIBAUDI (1996 : 26) définit l'approche configurationnelle comme une lecture des mécanismes historiques selon laquelle: "une variable isolée a un poids relatif qui ne se définit que dans son rapport avec d'autres variables, avec d'autres éléments. Il ne semble donc pas possible d'envisager un déterminisme rigide ou un fort mécanisme causal : les choix et les comportements (individuels ou collectifs) sont définis par une configuration d'éléments œuvrant à différents niveaux de la réalité et variant, dans leur force comme dans leur poids, selon leur disposition réciproque, selon les moments et les conjonctures historiques ». Cette approche, étroitement liée au courant microhistorique, se démarque notamment par son souci de restituer le rôle des configurations locales - c'est-à-dire la trame locale des relations et des appartenances sociales - dans l'explication des processus historiques globaux. 
En dépit de leur défaite face aux Français, les Tall, descendants d'El Hadj Omar, constituent une famille prestigieuse et influente au Soudan français $^{40}$. Ils forment un groupe d'aristocrates toucouleurs, anciens guerriers ou marabouts se réclamant d'une même figure historique et partisans d'un même mouvement religieux, la confrérie Tidjane. Cependant, la famille est divisée en branches rivales suite au conflit qui opposa les héritiers d'El Hadj Omar après sa mort. À l'époque coloniale, la famille Tall est ainsi éclatée sur un ensemble de localités ${ }^{41}$, anciennes capitales de l'empire oumarien, et sur lesquelles chaque branche des Tall tente de maintenir son emprise. Or, d'une localité à l'autre, l'attitude de la famille Tall, à l'égard du nouveau pouvoir colonial en général et de l'institution scolaire en particulier, varie considérablement. A Bandiagara, Aguibou Tall puis son fils Alfa Maki Tall (eux-mêmes respectivement fils et petit-fils d'El Hadj Omar Tall) sont nommés chef de la ville par les Français alors que ces derniers avaient jusque-là fait la guerre à l'empire oumarien. Dès la génération d'Aguibou Tall, une grande partie de la branche Tall de Bandiagara s'oriente vers l'École et trouve un emploi dans la fonction coloniale ${ }^{42}$. Après Aguibou, son fils Alfa Maki poursuit la politique de collaboration avec le nouveau pouvoir et choisit, à son tour, d'envoyer ses garçons à l'école. La plupart de ses fils ont été scolarisés dans les années 1910-1920, ils ont embrassé des carrières administratives ${ }^{43}$, et deux d'entre eux, Karamoko (son premier fils) et Seikou (son dernier fils), sont devenus instituteurs après avoir obtenu le diplôme de l'ENWP. La famille Tall de Bandiagara a ainsi fait le choix de s'engager dans la fonction coloniale pour perpétuer une influence menacée par la conquête.

À Nioro, la branche locale de la famille Tall n'a pas fait le même choix. Benjamin Soares (1999 : 206-207), qui a étudié l'histoire de la ville, souligne en effet le refus des Tall de Nioro d'envoyer leurs enfants à l'École française et cela jusqu'à une époque très récente. Ceux qui y sont recrutés de force comme Hady Tall, devenu aujourd'hui un membre éminent de la famille, n'y restent d'ailleurs pas longtemps ${ }^{44}$. Pour expliquer cette attitude

40. Pour l'histoire de cette famille à Bandiagara (mais aussi pour un compte rendu des querelles intestines à la famille Tall), voir BARRY (1993).

41. Les villes principales de résidence de la famille Tall au Soudan français sont alors Nioro, Ségou et Bandiagara.

42. Parmi les dix enfants d'Aguibou dont Marty fait mention en 1920, on trouve un télégraphiste, un sous-lieutenant, trois élèves de l'École française, deux chefs (Alfa Maki puis Tidiani) et deux commerçants. Par contre, un fils d'Aguibou a choisi la rébellion ouverte contre son père et contre les Français en menant une résistance armée dans le pays mossi jusqu'à sa mort en 1914 (MARTY 1921, t. 2 : 209).

43. Ainsi le frère aîné de Seikou Tall, de même mère que lui, a fait l'École PinetLaprade de Dakar d'où il est sorti ouvrier d'imprimerie (entretien avec Seikou Tall, ancien instituteur et fils d'Alfa Maki Tall, Ouagadougou, janvier 1998).

44. Selon SoAres (1999), Hady Tall a subi un recrutement forcé pendant deux ans à l'École française de Boghé (Mauritanie) où il est né alors que son père s'instruisait auprès d'un maître religieux de Boghé. Après avoir quitté l'École française, Hady a lui-même entrepris des études coraniques près de Kayes au Mali. 
de rejet, Soares émet l'hypothèse que les Tall de Nioro, qui vivent essentiellement des dons faits aux religieux, préfèrent s'investir dans l'enseignement islamique et revendiquent plus généralement une pratique religieuse exclusive de l'instruction occidentale. En cela, ils empruntent une voie très différente de la branche Tall de Bandiagara mais aussi d'une autre famille de responsables religieux présents à Nioro, les Kabalanke ou Kaba Jakhité. Les Kabalanke sont d'anciens clients des Tall qui ont su tirer parti de la présence française pour s'approprier le pouvoir à Nioro. À l'instar d'Alfa Maki Tall, Fodé Bajiné, chef de la famille Kaba Jakhité, est investi de la chefferie locale par les Français. Il favorise lui aussi l'envoi de ses enfants et ceux des clans associés à l'École française. Soares note que la famille des Kabalanke ainsi que la famille «associée » ${ }^{45}$ Maguiraga ont fourni une part importante des fonctionnaires africains originaires de la ville ${ }^{46}$. Nos propres recherches sur les Pontins originaires du cercle de Nioro confirment ces résultats. Lors d'un entretien avec l'un des fils de Fodé Maguiraga, ancien instituteur passé par Ponty et originaire de Nioro, celui-ci affirmait que sa famille a établi des alliances matrimoniales avec les Kaba Jakhité et que son père a été envoyé à l'école parce que les Kaba Jakhité y ont réussi et montré l'exemple en fournissant «des instituteurs, des interprètes et des chefs locaux ${ }^{47}$. Les Kabalanké n'en affichent pas moins leur statut de responsables religieux en faisant référence à la figure fondatrice de leur clan, l'érudit Alfa Umar Kaba Jakhité. Selon Soares (1999: 181), les Kabalanké défendent une conception de la communauté musulmane rendue compatible avec l'instruction occidentale à la différence de leurs rivaux, les Tall de Nioro.

Retourné à Nioro où il devint un responsable religieux assez réputé, il n'a jamais souhaité envoyer ses enfants à l'école.

45. Ces deux familles sont originaires du Boundou au Sénégal. Les Maguiraga semblent avoir suivi les Kabalanké après leur installation à Nioro (SOARES 1999).

46. «Early in the colonial period, many, if not all, of the employees of the colonial judicial system (judges and clerks) in the immediate area were selected from the most prominent families of religious specialists, the Kabalanke and the Semeya among the soninke, and from several Bida lineages from the "Moors" in Nioro » (SoARES 1999: 54). Nous avons également rencontré Benjamin Soares lors de son séjour d'études au Centre d'études africaines. Il nous a confirmé oralement cette idée d'un engagement très fort des familles Kaba Jakhité et Maguiraga dans la fonction coloniale puis dans l'administration malienne. Soares explique également la différence de comportements entre les Tall et les Kabalanké à Nioro par l'existence de conceptions différentes de l'identité musulmane à l'époque coloniale : «For Kabalanké, as for many others in the town, there was no contradiction between being a Muslim and having a secular education »... « For the Tall and their associates, on the other hand, their avoidance of secular education and many of the employment activities associated with such education allowed them to remain more firmly committed to the Tijjannya »... « Indeed for many of them, to be Muslim necessarily meant to be Tijani » (ibid.).

47. Entretien avec M. Maguiraga, fils de F. Maguiraga, ancien instituteur, Bamako, mai 1998. 
Schéma I. - Les Tall de Nioro et de Bandiagara : UN PORT OPPOSÉ À «L'ÉCOLE DU BLANC »

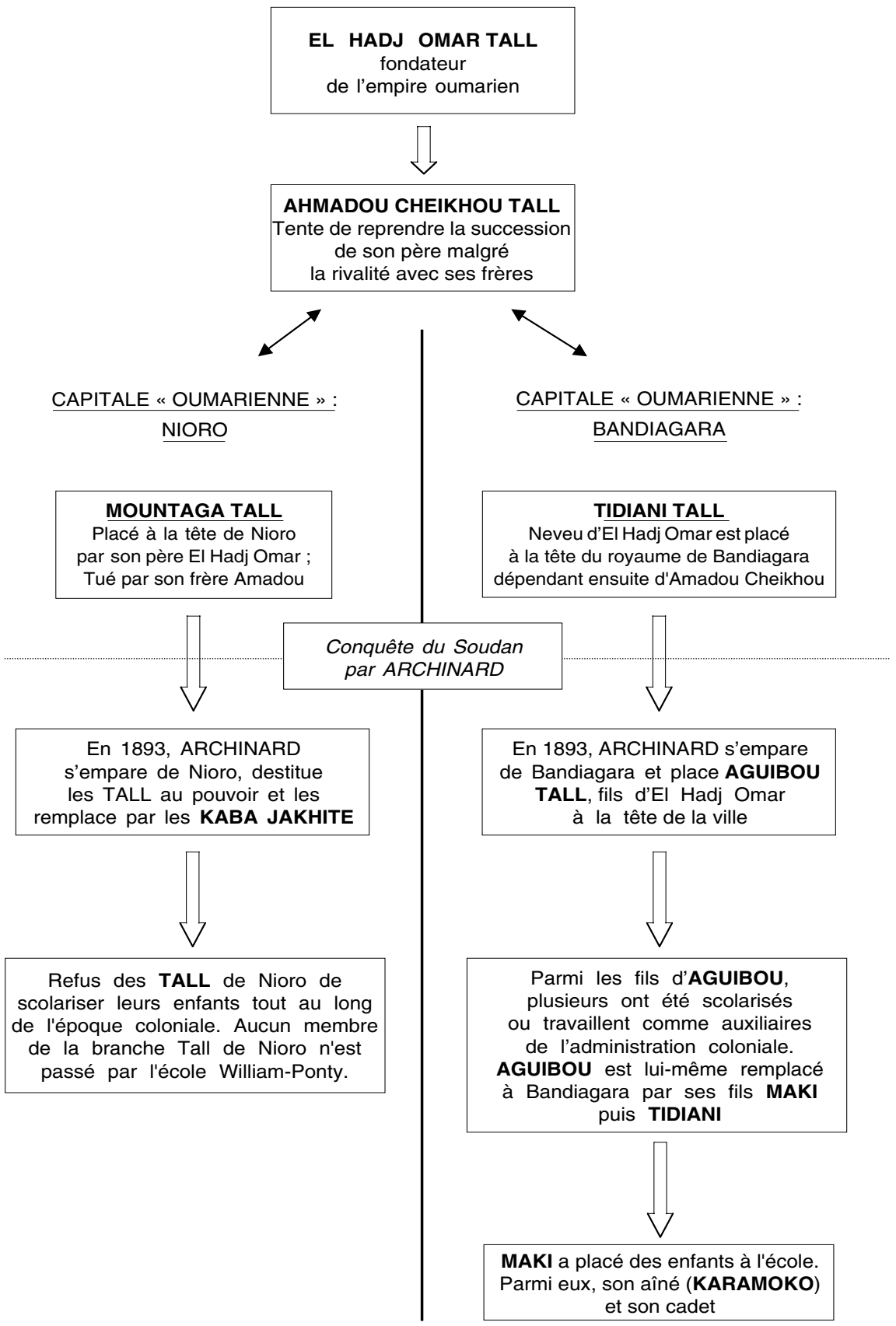

Source: Soares, 1999 ; Marty, 1921. 
Schéma II. - L'AsCEndance DE F. L. Touré

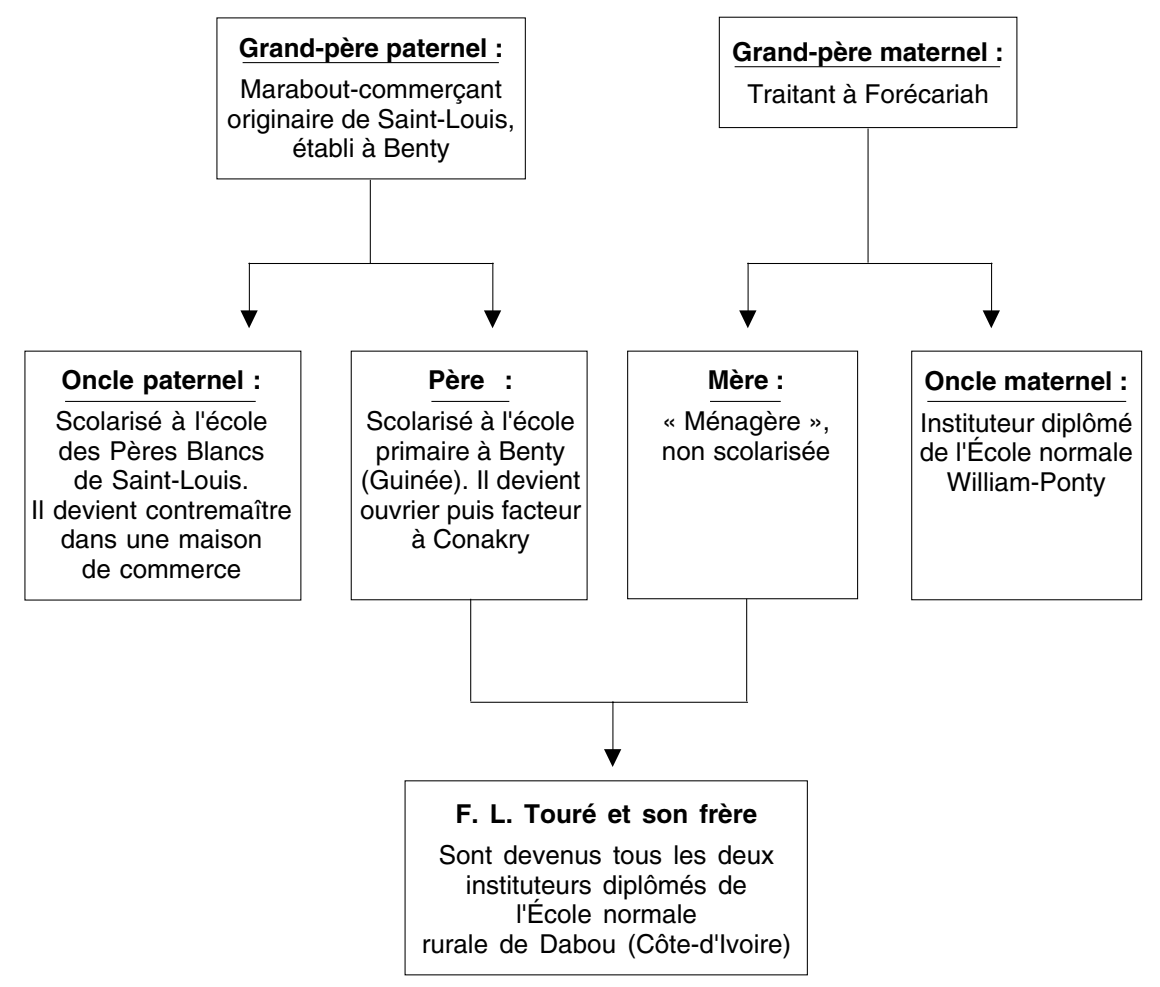

En définitive, une même famille d'aristocrates-guerriers et de religieux, les Tall, développe des stratégies différentes envers l'École en fonction de l'environnement dans lequel ses membres évoluent (voir tableau ci-dessus). À Nioro, les Tall ont été écartés par les Français qui leur ont préféré une autre famille, les Kaba Jakhité. À l'inverse de la branche Tall de Bandiagara, restée au pouvoir au début de la période coloniale, la branche Tall de Nioro n'a pas été en mesure ou n'a pas voulu développer une même stratégie de scolarisation précoce. Cet exemple souligne combien la trame locale des relations sociales, en partie recomposée lors de la conquête mais aussi largement dépendante de logiques précoloniales, influence les stratégies de scolarisation. Il ne s'agit pas pour autant de nier que l'islam a constitué un obstacle à la scolarisation dans de nombreuses régions d'Afrique de l'Ouest. Mais cette seule assertion ne permet pas de comprendre les itinéraires précoces de scolarisation que l'on observe au sein de milieux fortement islamisés. À l'inverse, en considérant l'appartenance religieuse comme une détermination dont le sens varie en fonction d'autres variables telles que les dynamiques historiques locales, on peut rendre compte des stratégies qui ont permis à certaines familles de concilier scolarisation et identité musulmane. 
Au-delà de l'islam, Il faut renoncer à concevoir le rôle de certaines variables comme l'appartenance ethnique, religieuse ou sociale sans les rattacher à un contexte qui leur donne sens. C'est également le cas pour « l'occidentalisation » du milieu d'origine, habituellement présenté comme un facteur favorable à une scolarisation précoce.

Le récit recueilli auprès de Fodé Lamine Touré, instituteur originaire de Conakry, montre ainsi que même dans un milieu familial dont le contact avec le monde occidental est ancien, l'École française ne constitue pas forcément la forme d'éducation la plus valorisée. La généalogie reproduite ciaprès montre combien la famille Touré de Conakry a établi, sur deux générations, des activités professionnelles liées à la présence coloniale et une stratégie de scolarisation des garçons de la famille.

Le schéma ci-dessus montre que la scolarisation est pratiquée par la famille de Fodé Lamine Touré aussi bien dans la branche paternelle que maternelle. À la génération des grands-parents tournée vers la traite a succédé celle des parents dont les activités sont liées à la fonction coloniale (un facteur, un instituteur) ou à l'emploi salarié (un contremaître). Pourtant, dans un premier temps, Fodé Lamine Touré et son frère aîné sont envoyés à l'École coranique et tenus à l'écart de l'École française. Dans son autobiographie, Fodé Lamine Touré (1997 : 56) se souvient comment lui était présentée l'École française :

«Déjà, à l'École coranique, nous formions deux catégories : ceux qui fréquentaient également l'École des Blancs, et nous autres, les traditionalistes. Les premiers que le karamoko [maître d'école coranique] affichait comme des pestiférés, faisaient l'objet d'un ostracisme évident, à ce point que l'École française était traitée de "dankacole", c'est-à-dire de "maudite école". Et quand le moment arrivait pour ces camarades de nous quitter pour leur nouvel établissement, c'était sous une pluie de quolibets, de sarcasmes et de malédiction qu'ils s'en allaient. »

Suite à la maladie mentale qui frappe son père, l'éducation du jeune Lamine est prise en main par son oncle paternel. Ce dernier, comme le montre le schéma précédent, a été scolarisé à Saint-Louis dans une école catholique. Il doit probablement son emploi de contremaître à cette formation scolaire. Pourtant, il refuse catégoriquement d'envoyer ses neveux à l'École française. Un vif débat l'oppose sur ce point à l'oncle maternel de Lamine, instituteur sorti de William-Ponty, qui souhaite que les enfants de sa sœur aillent à l'École française. Finalement, Lamine et son frère aîné, lassés des exactions dont ils sont victimes à l'École coranique, décident eux-mêmes de se rendre à l'École française sans en dire mot à leur oncle. Ce dernier, mis devant le fait accompli, finit par céder.

Le récit de Fodé Lamine Touré est intéressant à un double titre. D'abord, il indique que l'occidentalisation du milieu d'origine ne favorise pas forcément la scolarisation. Des formes concurrentes d'éducation, ici l'École coranique, persistent dans les milieux entrés précocement en contact avec les 
colonisateurs français. Ensuite, l'itinéraire de Fodé Lamine Touré souligne la fragilité des pratiques scolaires à l'époque coloniale. Le hasard semble en effet jouer un rôle important dans les itinéraires de scolarisation : que serait-il arrivé si Touré et son frère n'avaient pas eu la curiosité de voir «l'École du Blanc» le jour de la rentrée ? Que serait-il arrivé si, mis au courant plus tôt, l'oncle de Lamine Touré s'était opposé à leur scolarisation ? Mais en définitive, si le hasard semble aussi important dans ces récits de scolarisation, c'est surtout parce que l'École constitue encore en ce début de $\mathrm{XX}^{\mathrm{e}}$ siècle une institution neuve et inégalement intégrée par les sociétés africaines.

Les processus de scolarisation en Afrique de l'Ouest qui se mettent en place avant la période d'engouement et de massification scolaire de l'après-guerre obéissent à des logiques complexes dont l'analyse nécessite le recours à plusieurs registres de causalité. La proximité, géographique ou sociale, avec le colonisateur est indéniablement un facteur important de scolarisation mais ne peut rendre compte à elle seule de l'ensemble des logiques à l'œuvre. Outre le fait qu'elle est impuissante à éclairer certains itinéraires de scolarisation que l'on qualifierait à tort de marginaux, l'influence de l'occidentalisation se révèle extrêmement équivoque. Elle cache une pluralité de stratégies et de dynamiques qui ne prennent sens que restituées au sein des configurations spécifiques qui composent les contextes de scolarisation. Ce caractère «modulaire » ou «configurationnel» des déterminations s'étend à l'ensemble des variables qui participent au processus de scolarisation.

Dans une situation coloniale marquée par l'incertitude et la fragilité des nouvelles pratiques de reproduction, l'analyse doit en effet renoncer à vouloir dégager des pratiques sociales entièrement constituées et unifiées. Dans la plus grande partie de l'Afrique occidentale, l'École reste un « fait nouveau » qui n'a pas encore acquis la dimension ou l'épaisseur d'un « fait social ». En conséquence, les nouvelles pratiques qui émergent lentement pour répondre à cette institution encore inégalement intégrée par les sociétés locales traversent les groupes et les appartenances.

En tentant de rendre compte de la complexité du phénomène, notre recherche se perdrait-elle alors dans l'écheveau des processus historiques, incapable de fixer et d'évaluer de façon plus précise les registres de causalité ou d'attribuer des coefficients rassurants à leur impact? Tout au contraire, il nous semble que souligner la complexité, la discontinuité et la fragilité des processus de scolarisation à l'époque coloniale constitue un résultat positif. En montrant que la scolarisation obéit à des logiques complexes et incertaines, on comprend pourquoi le groupe des élites et des auxiliaires lettrés issus de la scolarisation constitue lui-même une strate extrêmement 
composite de par ses origines et de par les logiques de sa sélection. C'est un résultat important pour qui entend éclairer non seulement les voies de la sélection scolaire mais aussi comprendre l'émergence de nouvelles identités collectives dans l'Afrique contemporaine.

EHESS, Paris.

\section{BIBLIOGR APHIE}

Autra, R.

1956 «Historique de l'enseignement en AOF », Présence Africaine, 6: 68-86.

Ayandele, E. A.

1992 The Ijebu of Yorubaland, 1850-1950. Politics, Economy and Society, Ibadan, Heinemann Educational Books.

BARRY, B.

1993 Le royaume de Bandiagara, 1864-1893. Le pouvoir, le commerce et le Coran dans le Soudan nigérien au XIX $x^{e}$ siècle, Thèse de $3^{\mathrm{e}}$ cycle, Paris, EHESS.

BAYART, J.-F.

1989 L'État en Afrique. La politique du ventre, Paris, Fayard.

BonINI, N.

1996 Éducation non scolaire et école primaire : les conséquences d'une rencontre. Une étude anthropologique de la transmission du savoir chez les Maasai de Tanzanie, Thèse de Doctorat, Paris, EHESS.

Bouche, D.

1975 L'enseignement dans les territoires français de l'Afrique occidentale de 1817 à 1920, 2 tomes, Lille, Université de Lille III.

CAPelle, J.

1990 L'éducation en Afrique noire à la veille des indépendances, Paris, Karthala-ACCT.

Clignet, R.

1967 «Ethnicity, Social Differenciation and Secondary Schooling in West Africa », Cahiers d'Études africaines, VII (2), 26 : 360-378.

1993 «La demande d'éducation : aspects sociologiques», in P. Hugon, M. Gaud $\&$ M. Penoul (dir), «Crises de l'éducation en Afrique », Afrique contemporaine, 172 : 108-118. 
Clignet, R. \& Foster, P.

1966 The Fortunate Few : A Study of Secondary schools and Students in the Ivory Coast, Evanston, IL, Northwestern University Press.

ConkLin, A.

1997 A Mission to Civilize : The Republican Idea of Empire in France and West Africa, 1895-1930, Stanford, Stanford University Press.

COQuery-Vidrovitch, C. (dir.)

1992 L'Afrique occidentale au temps des Français. Colonisateurs et colonisés, c. 1860-1960, Paris, La Découverte.

Coulon, C.

1975 « Pouvoir oligarchique et mutations sociales et politiques au Fouta-Toro », in J.-L. Balans, C. Coulon \& J.-M. Gastellu, Autonomie locale et intégration nationale au Sénégal, Paris, A. Pedone : 23-80.

DÉSALMAND, P.

1983 Histoire de l'éducation en Côte-d'Ivoire des origines à la conférence de Brazzaville, Abidjan, CEDA.

DiOP, B.

1982 À Rebrousse-temps. Mémoires, t. 2, Paris, Présence africaine.

FosTer, P.

1965 Education and Social Change, Chicago, University of Chicago Press.

FOURCHARD, L.

2001 De la ville coloniale à la ville africaine. Espaces, pouvoirs et sociétés à Ouagadougou et à Bobo-Dioulasso (Haute-Volta). Fin XIX e siècle-1960, Paris, L'Harmattan.

GÉRARD, É.

1997 La tentation du savoir en Afrique. Politiques, mythes et stratégies d'éducation au Mali, Paris, Karthala-Orstom.

GRIBAUDI, M.

1996 «Échelle, pertinence, configuration », in J. Revel, Jeux d'échelles. La microanalyse à l'expérience, Paris, Gallimard-Éditions du Seuil : 113-139.

Guth, S.

1990 «L'école en Afrique noire francophone : une appropriation institutionnelle», Revue française de Pédagogie, 90 : 71-97.

JÉZÉQUEL, J. H.

2002 "Les mangeurs de craies». Socio-histoire d'une catégorie lettrée à l'époque coloniale. Les instituteurs diplômés de l'école normale William-Ponty, Doctorat, Paris, EHESS. 
JoHNSON, W. G.

1971 The Emergence of Black Politics in Senegal. The Struggle for Power in the Four Communes, 1900-1920, Stanford, Stanford University Press.

KHAYAR, I. H.

1976 «Le refus de l'école». Contribution à l'étude des problèmes de l'éducation chez les musulmans du Soudan (Tchad), Paris, Librairie d'Amérique et d'Orient.

KIPKORIR, B. E.

1973 « The Traditional Background to the Modern Kenyan African Elite : Kenya, 1890-1903 », in Third International Congress of Africanists, Addis Abeba, non publié.

LANGe, M.-F. \& Martin, J.-Y.

1995 «Les stratégies éducatives en Afrique. Le face à face États/sociétés », Cahiers des Sciences humaines, XXXI (3) : 563-574.

LATOUR, É. DE

1987 «Le Futur antérieur », in M. H. Piault (dir.), La colonisation : rupture ou parenthèse, Paris, L'Harmattan : 123-176.

MARTIN, J.-Y.

1972 «Sociologie de l'enseignement en Afrique noire », Cahiers internationaux de Sociologie, LIII : 337-362.

MARTY, P.

1921 Études sur l'Islam et les tribus du Soudan, t. 2, Paris, Leroux.

Moumouni, A.

1964 L'éducation en Afrique, Paris, Maspero.

NiANE, B.

1987 Des instituteurs aux énarques : l'École normale William-Ponty, l'École normale supérieure et l'École normale d'administration dans le processus de formation de la classe dirigeante au Sénégal, Paris, diplôme de l'EHESS.

Piault, M. H. (dir.)

1987 La colonisation : rupture ou parenthèse, Paris, L'Harmattan.

ReVel, J. (dir.)

1996 Jeux d'échelles. La micro-analyse à l'expérience, Paris, Gallimard-Éditions du Seuil.

RIVIÈRE, C.

1975 Dynamique de la stratification sociale en Guinée, Lille, Université Lille III.

Rosental, P. A.

1996 «Construire le "macro" par le "micro" : Frédérik Barth et la microstoria », in J. REVEL, op. cit. : 141-159. 
SABATIER, P.

1976 Educating a Colonial Elite. The William Ponty School and its Graduates, Chicago, Unpublished Ph. D.

1977 «"Elite Education" in French West Africa : The Era of Limits, 1903-1945 », International Journal of African Historical Studies, XI (2) : 247-266.

SADJI, A. B.

1997 Abdoulaye Sadji. Biographie, Paris, Présence africaine.

SOARES, B.

1999 The Spiritual Economy of Nioro du Sahel, Islamic Discourse and Practice in a Malian Religious Center, Evanston, Unpublished Ph. D.

Tibenderana, P. K.

1983 «The Emirs and the Spread of Western Education in Northern Nigeria, 1900$1946 »$, Journal of African History, 24 : 517-534.

TIREFORT, A.

1979 «Le bon temps». Approche de la société coloniale. Étude de cas : la communauté française en basse Côte-d'Ivoire pendant l'Entre-deux-guerres (19201941), 2 vols, Thèse de $3^{\text {e }}$ cycle, Paris, EHESS.

Touré, Fodé Lamine

1997 Une enfance africaine, t. 1, Conakry, Bibliothèque franco-guinéenne.

TRIAUD, J.-L.

1992 «L'Islam sous le régime colonial» in C. Coquery-VIDROvitch (dir.), L'Afrique occidentale au temps des Français. Colonisateurs et colonisés, c. 1860-1960, Paris, La Découverte : 141-155.

\section{RÉSUMÉ}

Cet article s'intéresse aux logiques qui président à la diffusion de l'École en Afrique de I'Ouest. II souligne d'abord les limites des interprétations univoques et linéaires du processus de scolarisation à l'époque coloniale. À partir d'une étude prosopographique centrée sur les diplômés de l'École Normale William-Ponty, il propose ensuite une approche plus attentive aux interprétations et aux appropriations différenciées de l'École par les sociétés locales. En effet, au temps colonial, l'École n'a pas encore acquis l'épaisseur d'un "fait social » auquel seraient liées des pratiques collectives déjà bien définies et partagées. Dès lors, il est nécessaire de développer une lecture historique attentive à l'impact différencié des facteurs de scolarisation en fonction des " configurations » dans lesquelles ils s'insèrent. Cet article montre finalement que dans la première moitié du $x^{\mathrm{e}}$ siècle, les itinéraires de scolarisation obéissent à des logiques complexes, fragiles et discontinues qui expliquent, par la suite, la grande diversité de l'élite lettrée ouest-africaine. 


\section{ABSTRACT}

A History of Desks, Pupils' Itineraries: For a "Configurational" Interpretation of Schooling during the Colonial Era. - What rationales have underlaid the spread of schooling in West Africa during the colonial era? The limits of unequivocal, linear interpretations are pointed out. On the basis of a prosopographic study of graduates from William-Ponty Teachers' Training College, an approach is proposed that pays attention to the differentiated ways local societies appropriated schools and interpreted education. During the colonial period, school was not yet a "social fact" linked to clearly defined and shared group practices. Hence, an attentive historical interpretation has to be developed of the differentiated impact of factors related to schooling as a function of the "configurations" of which it was a part. In the first half of the $20^{\text {th }}$ century, the "itineraries" of these graduates were based on complex, fragile, discontinuous rationales that account for the wide diversity of the educated elite in West Africa.

Mots-clés/Keywords : Afrique de I'Ouest, École normale William-Ponty, histoire, scolarisation/West Africa, William-Ponty Teachers' Training College, history, schooling. 